\title{
SIGMOID-TO-FLUX-ROPE TRANSITION LEADING TO A LOOP-LIKE CORONAL MASS EJECTION
}

\author{
Rui Liu ${ }^{1}$, Chang Liu ${ }^{1}$, Shuo Wang ${ }^{1}$, Na Deng ${ }^{1,2}$, and Haimin Wang ${ }^{1}$ \\ ${ }^{1}$ Space Weather Research Laboratory, Center for Solar-Terrestrial Research, NJIT, Newark, NJ 07102, USA; rui.liu@ njit.edu \\ 2 Physics and Astronomy Department, California State University Northridge, Northridge, CA 91330, USA \\ Received 2010 October 11; accepted 2010 November 1; published 2010 November 22
}

\begin{abstract}
Sigmoids are one of the most important precursor structures for solar eruptions. In this Letter, we study a sigmoid eruption on 2010 August 1 with EUV data obtained by the Atmospheric Imaging Assembly (AIA) on board the Solar Dynamic Observatory (SDO). In AIA $94 \AA$ (Fe XVIII; $6 \mathrm{MK})$, topological reconfiguration due to tether-cutting reconnection is unambiguously observed for the first time, i.e., two opposite J-shaped loops reconnect to form a continuous S-shaped loop, whose central portion is dipped and aligned along the magnetic polarity inversion line (PIL), and a compact loop crossing the PIL. A causal relationship between photospheric flows and coronal tethercutting reconnections is evidenced by the detection of persistent converging flows toward the PIL using line-of-sight magnetograms obtained by the Helioseismic and Magnetic Imager on board SDO. The S-shaped loop remains in quasi-equilibrium in the lower corona for about 50 minutes, with the central dipped portion rising slowly at $\sim 10 \mathrm{~km} \mathrm{~s}^{-1}$. The speed then increases to $\sim 60 \mathrm{~km} \mathrm{~s}^{-1}$ about 10 minutes prior to the onset of a GOES-class C3.2 flare, as the S-shaped loop speeds up its transformation into an arch-shaped loop, which eventually leads to a looplike coronal mass ejection. The AIA observations combined with $\mathrm{H} \alpha$ filtergrams as well as hard X-ray imaging and spectroscopy are consistent with most flare loops being formed by reconnection of the stretched legs of lesssheared J-shaped loops that envelopes the rising flux rope, in agreement with the standard tether-cutting scenario.
\end{abstract}

Key words: Sun: coronal mass ejections (CMEs) - Sun: filaments, prominences - Sun: flares

Online-only material: animations, color figures

\section{INTRODUCTION}

Coronal mass ejections (CMEs) and flares are responsible for the most energetic space weather phenomena, yet our knowledge of the driving mechanisms and the actual conditions leading to the eruption is limited. To achieve better understanding, we must identify the key aspects of eruptive structures that can provide diagnostic information for comparison with and incorporation into the models.

A distinctive set of structures that have been under special scrutiny are sigmoids (Rust \& Kumar 1996), which are forward or inverse S-shaped coronal loops seen often in soft X-ray (SXR) and sometimes in EUV (e.g., Liu et al. 2007) emission. They are often composed of two opposite J-like bundles of loops which collectively make an S-shape appearance (Canfield et al. 2007; McKenzie \& Canfield 2008). Sigmoidal regions are significantly more likely to be eruptive than non-sigmoidal regions (Hudson et al. 1998; Canfield et al. 1999; Glover et al. 2000). The eruption is usually followed by the formation of unsheared arcades or cusped loops, a process termed "sigmoid-to-arcade" evolution (e.g., Sterling et al. 2000; Moore et al. 2001; Pevtsov 2002; Gibson et al. 2002). In close spatial association with sigmoids are filaments (Pevtsov 2002), with which the central portion of the sigmoid is approximately aligned. The filament channel is known as a tracer of sheared field (Martin 1998), and the dense filament material is suggested to be supported either by a helically coiled field (Gibson \& Fan 2006, and references therein), or by a sheared arcade (e.g., Antiochos \& Klimchuk 1991), against the solar gravity.

It is appealing to associate sigmoids with kinked flux ropes (Rust \& Kumar 1994, 1996), whereas it remains controversial whether the observed sigmoids carry sufficient magnetic twist for the onset of the kink instability (Leamon et al. 2003; Leka et al. 2005). Titov \& Démoulin (1999) suggested that the transient sigmoidal brightening outlines magnetic structures with enhanced current density. This has been demonstrated in several MHD simulations, in which, for example, the sigmoid is identified either with separatrix surface associated with a baldpatch topology (Fan \& Gibson 2004) or with J-shaped field lines that pass through the vertical current sheet below a rising kink-unstable loop (Kliem et al. 2004).

Based on morphological changes of flaring structures, a physical picture for sigmoid eruption was proposed by Moore \& Labonte (1980) and further elaborated by Moore et al. (2001), in which the magnetic explosion is unleashed by the so-called tether-cutting reconnection, occurring between the inner legs of the two elbows of the sigmoid where they shear past each other under the filament. This reconnection produces a shorter low-lying loop across the polarity inversion line (PIL) and a longer twisted loop connecting the far ends of the sigmoid. The eruption begins when the twisted loop becomes unstable and distends the envelope field that overarches the sigmoid. The legs of the stretched envelope field subsequently reconnect back to form an arcade structure and the ejecting plasmoid escapes as a CME. Evidence for the tether-cutting model can also be found in recent observational studies (e.g., Wang 2006; Yurchyshyn et al. 2006; Liu et al. 2007). Liu et al. (2008) studied an EUV sigmoid, which is discontinuous at where it crosses the filament prior to the eruption, but becomes a single continuous structure lying above the filament at the eruption onset. This particular feature is at variance with Moore et al. (2001), in which the tether-cutting reconnection occurs below the filament.

The aforementioned observational studies, however, are often compromised by instrumental constraints, due to which some important aspects of the tether-cutting model remain ambiguous: (1) is the eruption initiated by tether cutting which leads to the rising of a flux rope or by the loss of equilibrium of the flux rope which later induces tether cutting below it? (2) Is the flux 

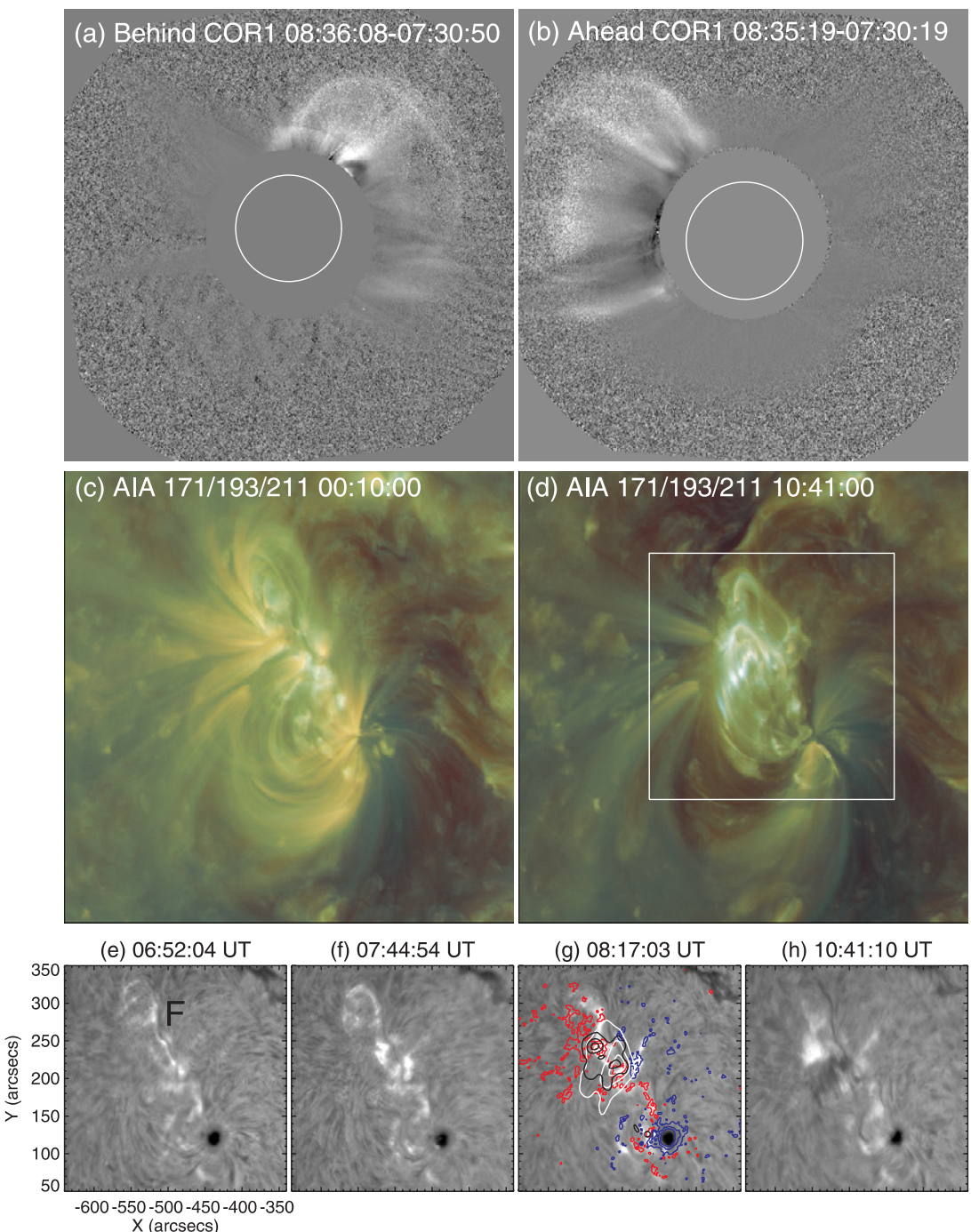

(g) 08:17:03 UT

(h) 10:41:10 UT

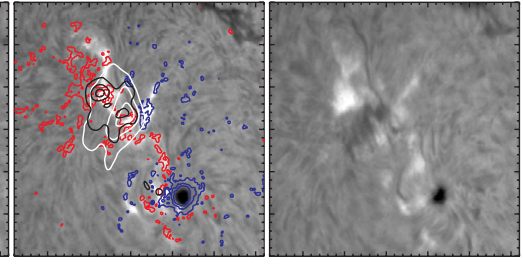

Figure 1. Overview of the sigmoid eruption on 2010 August 1. Panels (a) and (b) show the CME captured by the COR1 coronagraph on board STEREO. Panels (c) and (d) show a pre- and a post-eruption AIA composite image, respectively. The rectangle in Panel (d) indicates the field of view of KSO H $\alpha$ images in Panels (e)-(h). The $\mathrm{H} \alpha$ images are registered with the one at 08:17 UT (panel (g)), which is co-aligned with and overlaid by the HMI magnetogram taken at the same time. The contours levels are $\pm 100, \pm 500$, and $\pm 1000 \mathrm{G}$, with red (blue) color for positive (negative) polarity. A filament located along the PIL is labeled " $F$ " in panel (e). Panel (g) is also overlaid by RHESSI hard X-ray (HXR) sources integrated from 08:17 to 08:18 UT, at 3-9 keV (white) and at 12-25 keV (black). The contour levels are $50 \%, 70 \%$, and $90 \%$ of the maximum brightness.

(A color version of this figure is available in the online journal.)

rope pre-existent or formed by tether cutting? (3) Is the filament supported by the sheared legs of the sigmoid or by an embedded flux rope? (4) Does the sigmoid itself, or the overlying arcade, or a pre-existent flux rope, erupt as the CME?

With the launch of Solar Dynamic Observatory (SDO), we are now in a better position to address the above issues. Here, we investigate a sigmoid eruption observed by $S D O$, in which a twisted flux rope formed via tether cutting, and subsequently transformed into an arch-shaped loop distending the overlying field and resulting in a GOES-class C3.2 flare and a loop-like CME. In the rest of the Letter, observations and data analysis are presented in Section 2, important conclusions and remaining issues are summarized in Section 3.

\section{OBSERVATIONS AND DATA ANALYSIS}

\subsection{Instruments and Data Sets}

The sigmoid studied here erupted at about 08:00 UT on 2010 August 1, and was fully covered by Atmospheric Imaging
Assembly (AIA) and Helioseismic and Magnetic Imager (HMI) on board SDO (Figures 1(c) and (d)), as well as by the $\mathrm{H} \alpha$ telescope at the Kanzelhöhe Solar Observatory (KSO; Figures 1(e)-(h)) starting from 06:52 UT. The subsequent CME was detected by the COR1 coronagraph on board both the Ahead and Behind satellites of the Solar Terrestrial Relations Observatory (STEREO; Figures 1(a) and (b)), which were separated by about $150 \mathrm{deg}$. The flare associated with the CME was detected by the Reuven Ramaty High-Energy Solar Spectroscopic Imager (RHESS; Lin et al. 2002).

AIA takes multi-wavelength images at 1 .'2 resolution and 12 s cadence. To study flaring plasma, we focus on the $94 \AA$ filter (Fe XVIII; $\log T=6.8$ ) as well as the $131 \AA$ A filter which covers Fe VIII $(\log T=5.6), \mathrm{Fe}$ XX $(\log T=7.1)$, and Fe XXIII $(\log T=7.2)$. To enhance signal-to-noise ratio, we add every five $94 \AA$ images to yield new images at effectively 1 minute cadence. Other filters in $171 \AA$ (Fe IX; $\log T=5.8), 193 \AA$ (Fe XII; $\log T=6.1), 211 \AA$ (Fe xIV; $\log T=6.3$ ), and $335 \AA$ (Fe XvI; $\log T=6.4$ ) provide a multi-thermal perspective. The 


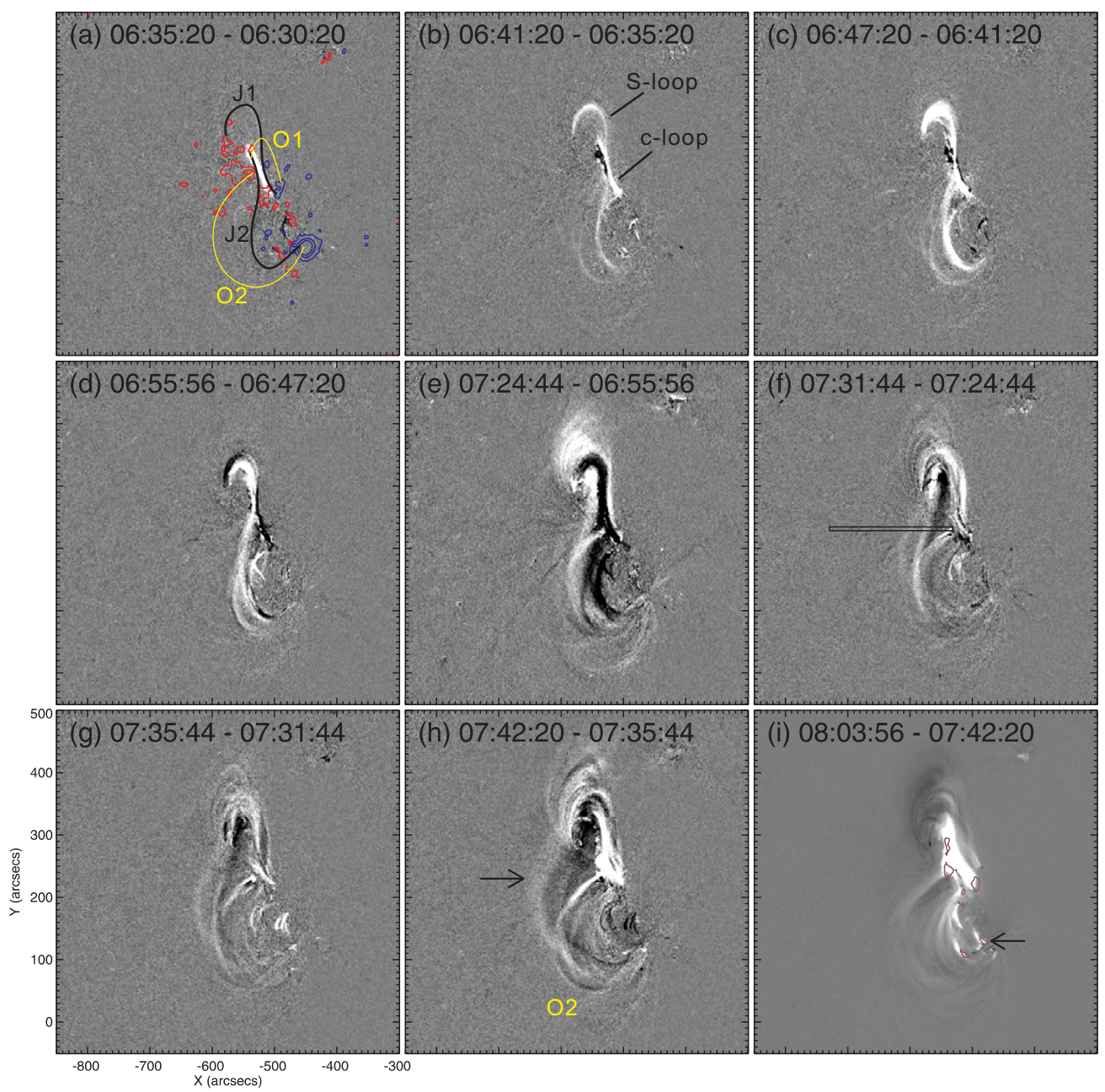

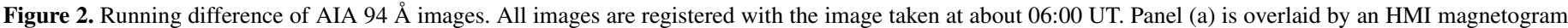

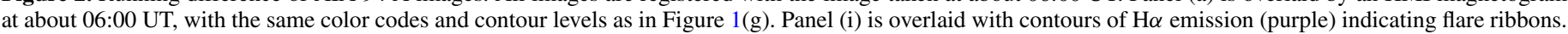
(An animation and a color version of this figure are available in the online journal.)

$193 \AA$ band is also sensitive to 20 MK plasma (Fe XXIV). HMI observes the Sun at $6173 \AA$ at $1^{\prime \prime}$ resolution and has begun to publish line-of-sight magnetograms at $45 \mathrm{~s}$ cadence with a precision of $10 \mathrm{G}$.

\subsection{The Formation of the Flux Rope}

The sigmoid eruption conforms to the classical "sigmoid-toarcade" transformation. Figure 1(c) shows the sigmoid structure observed $8 \mathrm{hr}$ before the eruption, which consisted of two bundles of J-shaped loops. The arcade structure after the eruption is shown in Figure 1(d). The detailed transformation is presented in Figure 2 as the running difference of AIA $94 \AA$ images. At 06:35 UT (Figure 2(a)), a brightening structure appeared in the center of the active region, which seems to be a low-lying, compact loop (hereafter c-loop) across the PIL. About 5 minutes later, an inverse S-shaped loop (hereafter S-loop) started to glow, whose central portion was apparently dipped, aligned along the PIL, and crossed the c-loop (Figure 2(b)). The S-loop axis is apparently twisted by about half a turn (referred to also as flux rope hereafter), but the individual field line could be twisted more than that.

The c-loop was soon darkened in the running difference image (Figure 2(d)) and became visible by 06:55 UT in cooler AIA filters such as $335 \AA$ and $211 \AA$. The S-loop can only be clearly seen in AIA $94 \AA$ throughout its lifetime. Its temperature is therefore well constrained, i.e., about $6 \mathrm{MK}$. Since both the S-loop and c-loop were newly formed and of high temperature, we suggest that they were the product of the reconnection between two J-shaped loops (hereafter J-loops) as illustrated in Figure 2(a) (labeled "J1" and "J2"), same as the tether-cutting reconnection envisaged by Moore et al. (2001). However, the cooling of the c-loop and the concurrent detachment of the S-loop with it suggest the halt of the reconnection by then. The fact that the c-loop brightened before the S-loop formed excludes the possibility that the reconnection was induced by the flux rope. Despite the heating observed in AIA, the amount of the energy released by tether cutting is below the detection threshold of both GOES and RHESSI, since the only flare detected at that time was above the southeast limb (GOES-class B3.3; see the inset of Figure 3(c)). This concurs with MHD simulations (e.g., Aulanier et al. 2010).

To investigate the role of photospheric flows in the formation of the flux rope, we use a series of HMI magnetograms at 12 minute cadence from 00:00 UT to 12:00 UT. The average flow field calculated by the differential affine velocity estimator (DAVE; Schuck 2005) is shown in Figure 4. The most visible flow pattern is the radial outflows in the sunspot penumbra (left panel), known as moat flows. Apart from that, one can see persistent converging motions toward, and 

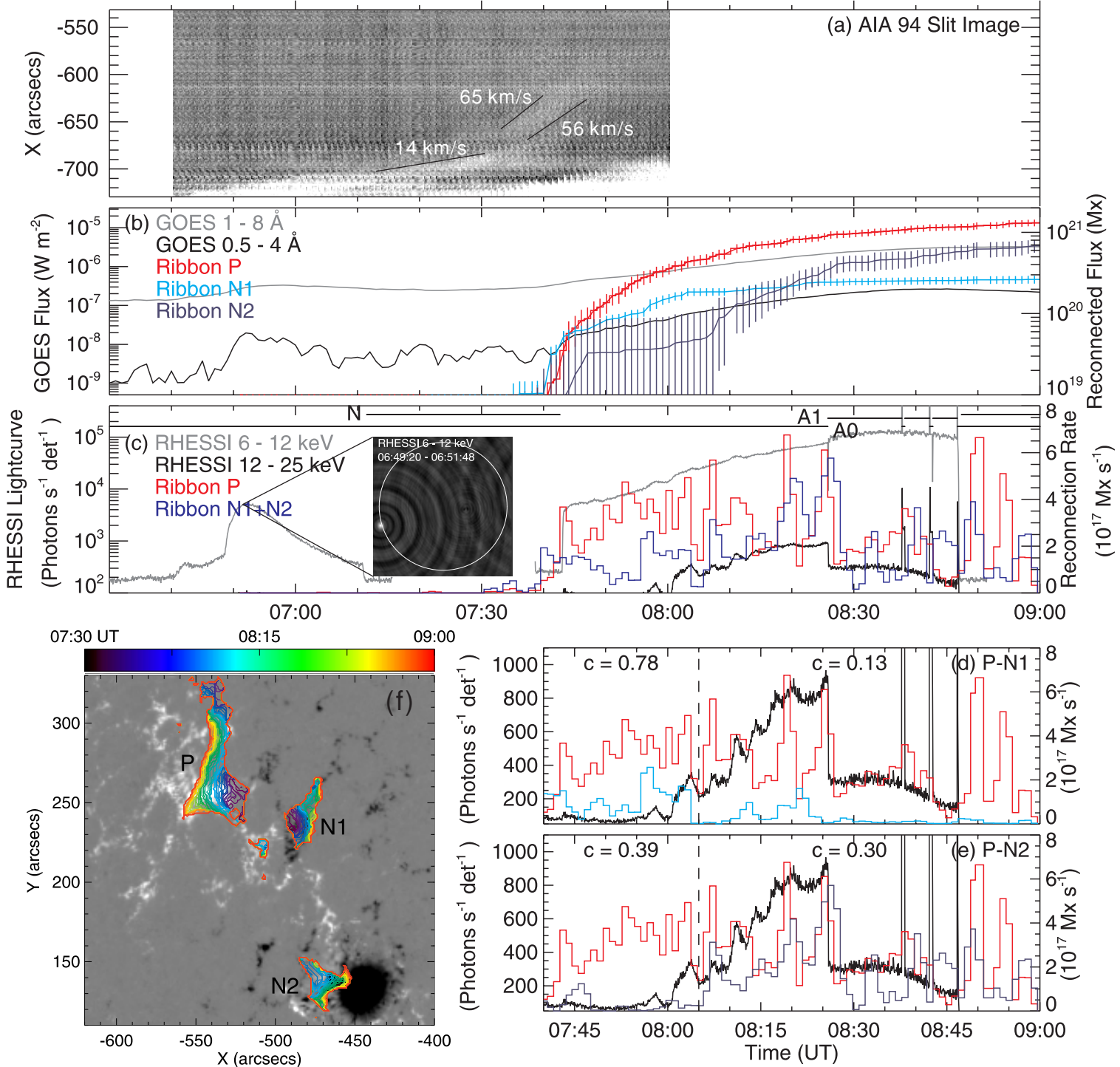

Figure 3. Time evolution of the sigmoid eruption in various wavelengths. In panel (c), the bars at the top indicate RHESSI eclipse ("N"), and the attenuator states, "A1" and "A0," respectively. The inset is a RHESSI back-projection image at 6-12 keV showing the B3.3 flare location. The correlation coefficients between Ribbons $\mathrm{P}$ and N1 before and after 08:05 UT (indicated by dashed line) are shown at the top of panel (d), and those between Ribbons P and N2 are shown in panel (e). In panel (f), color-coded contours display the time evolution of $\mathrm{H} \alpha$ ribbons, overlaid on an HMI magnetogram.

(A color version of this figure is available in the online journal.)

less significant shear motions along, the PIL (right panel). Magnetic elements of positive polarity (white patches and red contours) show stronger converging motions than those of negative polarity (black patches and blue contours). The overall flow pattern is independently confirmed by local correlation tracking (November \& Simon 1988). Flux cancellation can be seen in the animation of magnetograms, when the white patches move into black ones. From the left panel of Figure 4, one can also see that the c-loop is clearly associated with the converging flows, which supports the conjecture of Moore et al. (2001). Formation and eruption of flux ropes driven by converging flows have also been found in three-dimensional MHD simulations (e.g., Amari et al. 2003; Aulanier et al. 2010).

\subsection{The Eruption}

In Figure 3(a), we present the chronological observation of the S-loop through a slit across its dipped portion (Figure 2(f)). This slit "image" is composed of strips cut from the AIA $94 \AA$ base-difference images (subtracted by the image at 06:00 UT). The projected speed of the rising flux rope can then be estimated from the brightening patterns. Figures 2 and 3(a) together show that after its formation, the S-loop began to rise slowly at $\sim 10 \mathrm{~km} \mathrm{~s}^{-1}$, driven presumably by the upward-pointing magnetic tension force at the dip. The rising speed then increased to $\sim 60 \mathrm{~km} \mathrm{~s}^{-1}$ at about 07:30 UT (Figure 3(a)), 10 minutes prior to the C3.2 flare (Figure 3(b)). At the flare onset (about 07:42 UT; Figure 3(b)), the twisted flux rope had transformed into an arch-shaped loop (Figure 2(h), marked by an arrow). Snapshots of the rising flux rope may have been captured previously (Moore et al. 2001; McKenzie \& Canfield 2008). This rising and inflating loop became too diffused to be seen after 07:50 UT, but only minutes later a CME with a similar loop morphology and loop orientation (Figures 1(a) and (b)) was observed first by the STEREO Ahead Satellite from 07:55 UT onward, and then by the Behind satellite from 08:11 UT onward. No high-density filament material (bright core) can be seen inside the CME and the filament lying along the PIL (see 


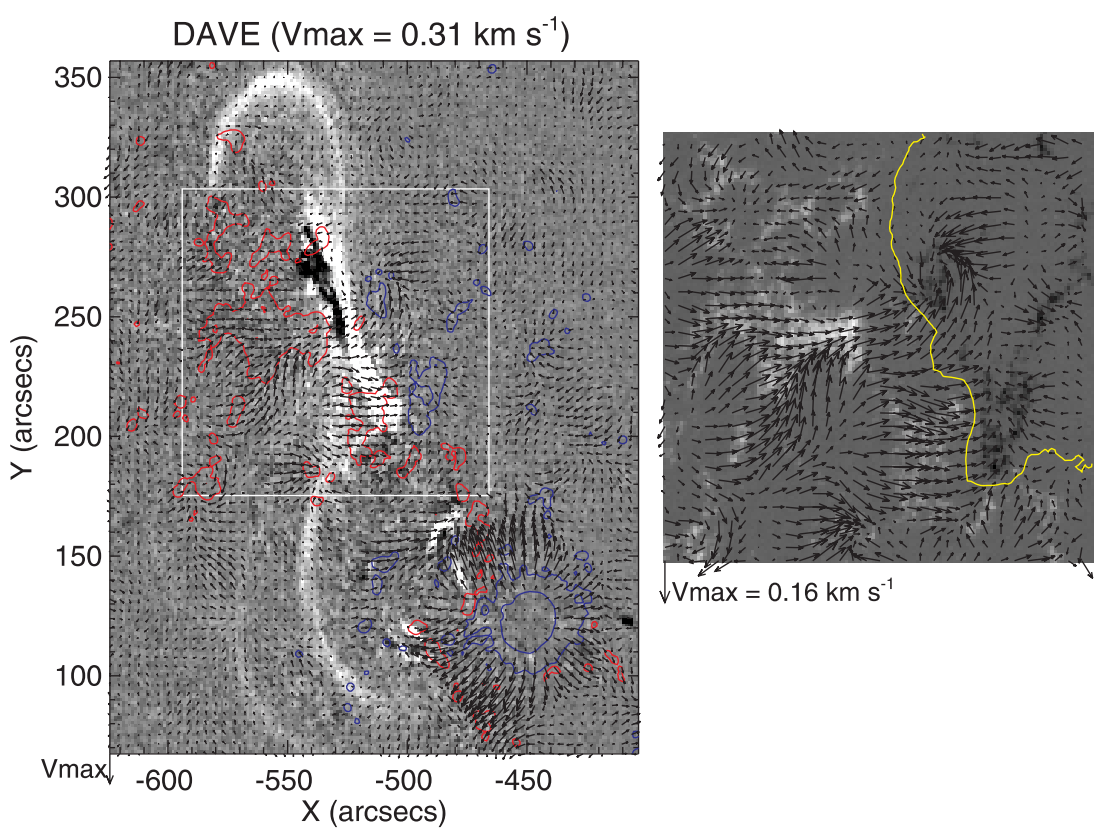

Figure 4. Flow field calculated with the DAVE method. Figure 2(b) is adopted as the background of the left panel. Contours levels are \pm 100 and $\pm 500 \mathrm{G}$, given by the HMI magnetogram at 06:00 UT (background of the right panel), to which all other magnetograms used for the calculation are registered. The rectangle in the left panel indicates the field of view of the right panel, in which the yellow contour indicates the PIL.

(An animation and a color version of this figure are available in the online journal.)

Figures 1(e)-(h); labeled "F" in Figure 1(e)) remained largely intact throughout the eruptive process. Hence, the tether-cutting reconnection must have taken place above the filament (see also Pevtsov 2002; Liu et al. 2007, 2008).

With the onset of the C3.2 flare, flaring loops which were less sheared than the c-loop became visible in the central region (Figures 2(h) and (i)). We suggest that the flaring is due to reconnection driven by the rising flux rope which stretched the loops overlying the central region and resulted in the formation of a current sheet underneath, similar to the schematic diagram in Moore et al. (2001, their Figure 1). In Figure 2(a), such an overlying loop is illustrated in yellow (labeled "O1"). Note, however, that $\mathrm{O} 1$ was not visible in any AIA filter prior to the flare. In fact, coronal loops arched over the rising flux rope were similar in magnetic connectivity to J1 and J2 in Figure 2(a), except that they were located higher and less sheared. Such an overlying loop is illustrated in yellow in Figure 2(a) (labeled "O2"). One can see that a group of loops similar to $\mathrm{O} 2$ were pushed upward by the rising flux rope, exhibiting a bright outer rim and a dark inner rim in the running difference image (Figure 2(h)). At 08:03 UT, a brightening ribbon in $\mathrm{H} \alpha$ became visible near the sunspot, cospatial with a brightening front moving westward in AIA $94 \AA$ (Figure 2(i), marked by an arrow). We suggest that from that time onward, the reconnection was due to the stretch of $\mathrm{O} 2$.

This interpretation is supported by $\mathrm{H} \alpha$ observations, in which three flare ribbons are identified and referred to as Ribbons $\mathrm{P}$, $\mathrm{N} 1$, and $\mathrm{N} 2$ hereafter (Figure 3(f)), with $\mathrm{P}$ for positive and $\mathrm{N}$ for negative polarity. In Figure 3(f) color-coded contours display the time evolution of $\mathrm{H} \alpha$ ribbons, overlaid on an HMI magnetogram to which $\mathrm{H} \alpha$ images are co-aligned. Obviously Ribbons $\mathrm{P}$ and $\mathrm{N} 1$ are connected by loops like $\mathrm{O} 1$, while $\mathrm{P}$ and $\mathrm{N} 2$ by loops like $\mathrm{O} 2$. We calculated the reconnection rate and the reconnected flux following Qiu \& Yurchyshyn (2005). The reconnection rate is defined as the change rate of the magnetic flux passing through the newly brightened flare area, and its time integration gives the reconnected flux. The results are shown in Figures 3(b)-(e). One can see that the reconnected flux at Ribbon N1 became flattened after about 08:05 UT, when the reconnection flux at Ribbon N2 started to pick up. Accordingly, the reconnection rate at Ribbon N2 dominated over that at Ribbon N1 after 08:05 UT (Figures 3(b)-(e)). Moreover, before 08:05 UT, Ribbon P was better correlated with Ribbon N1 in terms of the reconnection rate, while after 08:05 UT, it was better correlated with Ribbon N2 (Figures 3(d) and (e)). About 2 hr later, post-flare loops began to appear in $\mathrm{H} \alpha$ as absorption features (Figure 1(h)). Like in EUV (Figure 1(d)), the $\mathrm{H} \alpha$ post-flare loops were oriented in a northeast-southwest direction, rather than the east-west direction crossing the filament, which also indicates that the majority of the flare loops connected Ribbons P to N2, rather than Ribbons P to N1.

RHESSI provides additional diagnostic information on the flare loops. A hard X-ray (HXR) spectrum integrated from 08:17 UT to 08:18 UT is shown in Figure 5(i) as an example. It can be well fitted with an isothermal component of $12 \mathrm{MK}$ (orange color) plus a power-law component with $\gamma \simeq 5$ above $11 \mathrm{keV}$ up to $25 \mathrm{keV}$ (green). A Gaussian line (purple) is adopted to fit the iron-line complex peaking at $\sim 6.7 \mathrm{keV}$. The HXR sources reconstructed with the CLEAN algorithm are shown as contours in Figure 5(e). One can see that the thermal source (3-9 keV; orange contours) is cospatial with the brightening in hot AIA filters, $94 \AA$ (Figure 5(g)) and $131 \AA$ (Figure 5(h)). The nonthermal component (12-25 keV; green contours) exhibits two strong sources: one is apparently associated with Ribbon P (Source 1), and the other, which is also registered with positive polarity when projected onto the magnetogram (see also Figure $1(\mathrm{~g})$ ), is cospatial with the thermal source (Source 2). Hence, we conclude that Source 1 is a footpoint source and Source 2 is a looptop source. There is an additional weak source (Source 3) cospatial with Ribbon N2 (Figure 1(g)), which appears to be the footpoint conjugate to Source 1. Before 08:05 UT, only Source 1 was visible (not shown). We 

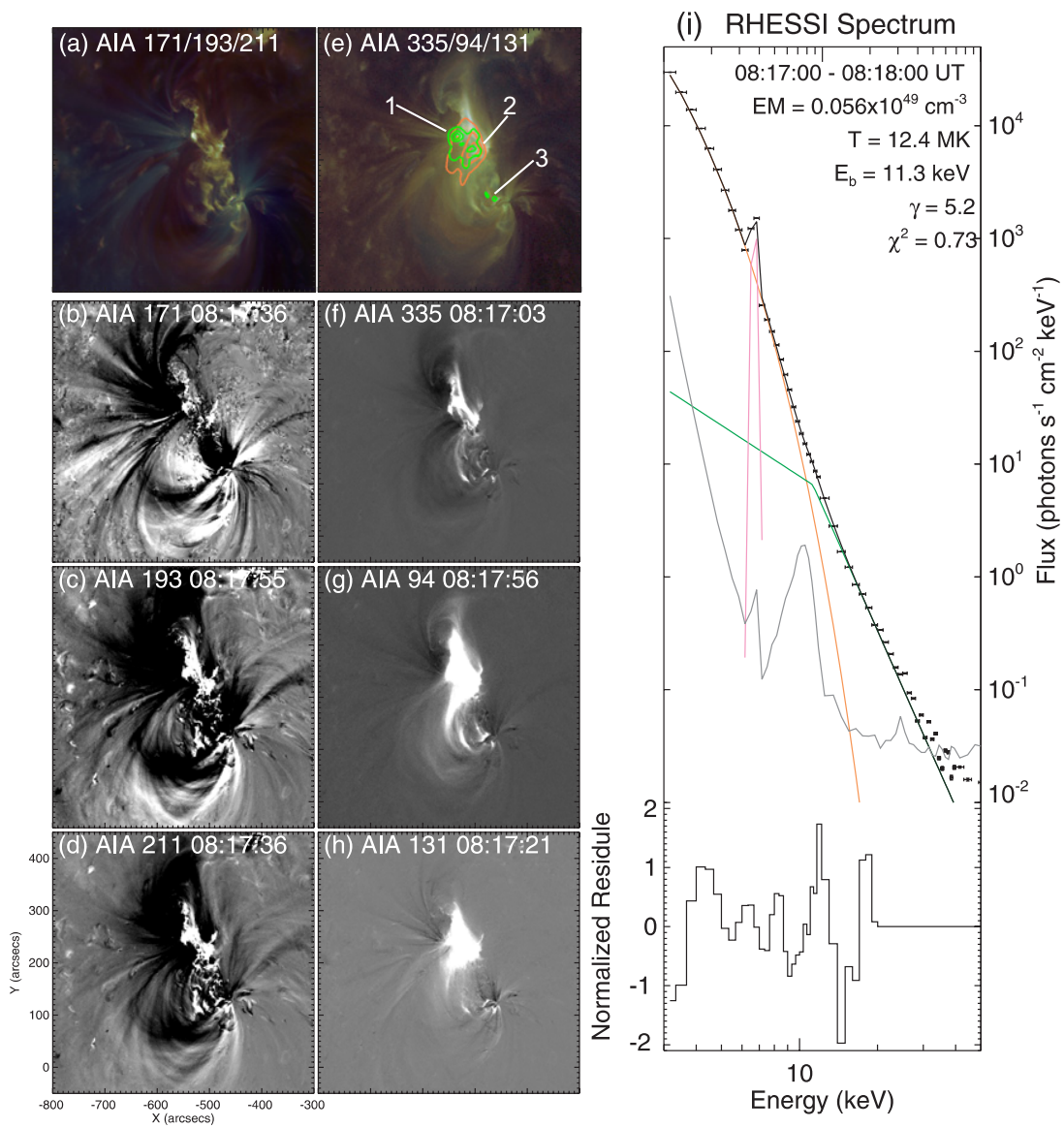

Figure 5. Flare observed by AIA and RHESSI. Panels (a) and (e) show composite images of cold filters (171 $\AA, 193 \AA$, and $211 \AA$ ) and hot filters ( $335 \AA$, $94 \AA$, and $131 \AA$ ), respectively. Panels (b)-(d) and (f)-(h) show the base-difference image of each individual filter (subtracted by an image at about 06:00 UT). Panel (e) is overlaid by the same HXR sources as in Figure 1(g), with three 12-25 keV sources being marked. Panel (i) shows a RHESSI spectrum, with fit residuals plotted at the bottom (see the text for spectral fitting details).

(A color version of this figure is available in the online journal.)

suspect that its conjugate footpoint at Ribbon N1 is too weak to be detected with RHESSI's limited dynamic range $(\sim 1: 10)$.

In addition, significant dimmings can be seen in cold AIA filters (Figures 5(b)-(d)), but not in hot filters (Figures 5(f)-(h)). This implies that coronal dimming is not only an effect of density depletion with the cold envelope filed being opened up, but also a temperature effect with plasmas in the core region being heated to high temperatures.

\section{DISCUSSION AND CONCLUSION}

The sigmoid eruption on 2010 August 1 evolved clearly in a sequential manner, which provides us an opportunity to address several controversial issues in solar physics.

First of all, the observation verifies, for the first time, that the topological reconfiguration due to tether-cutting reconnection does exist in the solar corona, i.e., two opposite J-shaped loops reconnect to form a continuous S-shaped loop and a compact low-lying loop (Figure 2). A causal relationship between photospheric flows and coronal tether-cutting reconnections is also evidenced by the detection of persistent converging flows toward the PIL (Figure 4). On the other hand, the observation that the filament was left intact during the sigmoid eruption (Figure 1), which is at variance with what the tether-cutting model predicts, calls for further investigation on how filaments are embedded in sigmoids.
Second, the observation that the S-shaped loop continuously transformed into an arch-shaped loop (Figure 2) and then erupted as a loop-like CME (Figure 1) shed light on the origin of interplanetary magnetic clouds, which possess rotating magnetic fields (Burlaga et al. 1981). It also makes it difficult for one to argue that the observed S-shaped brightening is due to current density enhancement below a dynamic flux rope.

Third, the observations that the tether-cutting reconnection was not detected as a flare and that the newly formed flux rope remained in quasi-equilibrium in the lower corona for about 50 minutes (Figures 2 and 3)) argue strongly for the existence of flux ropes prior to solar eruptions (see also Tripathi et al. 2009; Green \& Kliem 2009). We suggest that there may exist a continuum distribution of the lifetime of "quiescent" flux ropes in the corona from minutes to hours, or even to days, depending on the amount of magnetic twist possessed by the flux rope and the strength of magnetic confinement imposed by the surroundings.

Finally, we infer from the observations that most flare loops were formed by reconnection of the stretched legs of lesssheared J-shaped loops that enveloped the rising flux rope (Figures 2,3, and 5). The reconnection apparently began in the sheared field above the filament so that some of the sigmoidal field was trapped under the flare arcade, consistent with the active region remaining sigmoidal during and after the eruption (e.g., Pevtsov 2002; McKenzie \& Canfield 2008). 
$S D O$ is a mission for NASA's Living With a Star (LWS) Program. KSO $\mathrm{H} \alpha$ data are provided through the Global H-alpha Network operated by NJIT. The authors thank P. Schuck for providing the DAVE code. R.L., C.L., S.W., and H.W were supported by NASA grants NNX08-AJ23G and NNX08AQ90G, and by NSF grants ATM-0849453 and ATM-0819662. N.D. was supported by NASA grant NNX08AQ32G.

\section{REFERENCES}

Amari, T., Luciani, J. F., Aly, J. J., Mikic, Z., \& Linker, J. 2003, ApJ, 585 1073

Antiochos, S. K., \& Klimchuk, J. A. 1991, ApJ, 378, 372

Aulanier, G., Török, T., Démoulin, P., \& DeLuca, E. E. 2010, ApJ, 708, 314

Burlaga, L., Sittler, E., Mariani, F., \& Schwenn, R. 1981, J. Geophys. Res., 86, 6673

Canfield, R. C., Hudson, H. S., \& McKenzie, D. E. 1999, Geophys. Res. Lett., 26,627

Canfield, R. C., Kazachenko, M. D., Acton, L. W., Mackay, D. H., Son, J., \& Freeman, T. L. 2007, ApJ, 671, L81

Fan, Y., \& Gibson, S. E. 2004, ApJ, 609, 1123

Gibson, S. E., \& Fan, Y. 2006, J. Geophys. Res., 111, A12103

Gibson, S. E., et al. 2002, ApJ, 574, 1021

Glover, A., Ranns, N. D. R., Harra, L. K., \& Culhane, J. L. 2000, Geophys. Res. Lett., 27, 2161

Green, L. M., \& Kliem, B. 2009, ApJ, 700, L83
Hudson, H. S., Lemen, J. R., St. Cyr, O. C., Sterling, A. C., \& Webb, D. F. 1998, Geophys. Res. Lett., 25, 2481

Kliem, B., Titov, V. S., \& Török, T. 2004, A\&A, 413, L23

Leamon, R. J., Canfield, R. C., Blehm, Z., \& Pevtsov, A. A. 2003, ApJ, 596, L255

Leka, K. D., Fan, Y., \& Barnes, G. 2005, ApJ, 626, 1091

Lin, R. P., et al. 2002, Sol. Phys., 210, 3

Liu, C., Lee, J., Yurchyshyn, V., Deng, N., Cho, K., Karlický, M., \& Wang, H. 2007, ApJ, 669, 1372

Liu, R., Gilbert, H. R., Alexander, D., \& Su, Y. 2008, ApJ, 680, 1508

Martin, S. F. 1998, Sol. Phys., 182, 107

McKenzie, D. E., \& Canfield, R. C. 2008, A\&A, 481, L65

Moore, R. L., \& Labonte, B. J. 1980, in IAU Symp. 91, Solar and Interplanetary Dynamics, ed. M. Dryer \& E. Tandberg-Hanssen (Cambridge: Cambridge Univ. Press), 207

Moore, R. L., Sterling, A. C., Hudson, H. S., \& Lemen, J. R. 2001, ApJ, 552, 833

November, L. J., \& Simon, G. W. 1988, ApJ, 333, 427

Pevtsov, A. A. 2002, Sol. Phys., 207, 111

Qiu, J., \& Yurchyshyn, V. B. 2005, ApJ, 634, L121

Rust, D. M., \& Kumar, A. 1994, Sol. Phys., 155, 69

Rust, D. M., \& Kumar, A. 1996, ApJ, 464, L199

Schuck, P. W. 2005, ApJ, 632, L53

Sterling, A. C., Hudson, H. S., Thompson, B. J., \& Zarro, D. M. 2000, ApJ, 532, 628

Titov, V. S., \& Démoulin, P. 1999, A\&A, 351, 707

Tripathi, D., Kliem, B., Mason, H. E., Young, P. R., \& Green, L. M. 2009, ApJ, 698, L27

Wang, H. 2006, ApJ, 649, 490

Yurchyshyn, V., Liu, C., Abramenko, V., \& Krall, J. 2006, Sol. Phys., 239, 317 